MATEC Web of Conferences 34, 04004 (2015)

DOI: $10.1051 /$ matec conf/ 20153404004

(c) Owned by the authors, published by EDP Sciences, 2015

\title{
Optimized direct inverse control to control altitude of a small helicopter
}

\author{
Herwin Suprijono ${ }^{1,2, a}$, Wahidin Wahab ${ }^{1}$ and Benyamin Kusumoputro ${ }^{1}$ \\ ${ }^{1}$ Universitas Indonesia, in Depok, Indonesia \\ ${ }^{2}$ on leave from Universitas Dian Nuswantoro, in Semarang, Indonesia
}

\begin{abstract}
In this paper, an optimization of a direct inverse control (DIC) algorithm is discussed. The DIC algorithm is constructed by using a neural network that was trained to find the mathematical inverse model of the plant. In the case of a DIC to control a small helicopter as a UAV in our case, it is required to collect the flight experiment data, such as the PWM signal into servo motors, and the flight output such as the pitch, roll, yaw and the altitude. By using a real small helicopter TREX 450, the neural network based DIC model was performed with an acceptable of error, however, in order to have a better performance, an optimized neural network DIC model is proposed by retraining the neural networks DIC model using a new data generated from a determined optimal reference pathway. The experiment results show that the optimized neural networks DIC model have a better performance with lower total error rate compare with that of the un-optimized neural networks DIC model.
\end{abstract}

\section{Introduction}

In general an Unmanned Aerial Vehicle (UAV) is an unmanned aircraft that is controlled automatically using a predetermined algorithm such that the aircraft can maintain its position or speed. The UAV can be divided into two categories, a fixed-wing UAV and a rotor wing UAV. Fixed-wing UAV requires a long runway for takeoff and landing and less agile in maneuvering, while a nonfixed wing or a rotary wing UAV has the advantage of a vertical take-off and landing, and hovering. As the consequence, the rotary wing UAV does not require a long runway and has the ability to agile-maneuvers to reach the difficult terrain conditions. Some UAV may controlled remotely by a pilot from a ground station to do maneuvers for specified purpose; however, we would like to control the maneuvers of the UAV in an autonomous mode. For that purpose, we constructed an UAV using the single rotor platform or better known as Single Rotor Unmanned Aerial Vehicle (RUAV) using a small-scale helicopter and a neural network based controller for autonomous movement of the helicopter.

Recently, RUAV is not only used just in the military tasks force but also in many areas of civil applications, such as for mapping, search and rescue (SAR) and surveillance. RUAV is capable of carrying out a dangerous task with lower cost and risk for human life, such as working in the condition of areas that were exposed to nuclear radiation, or disaster places that is difficult to be reached. However, a small-scale RUAV has a complex dynamic system with characteristics of Multiple Input Multiple Output (MIMO), under actuated, nonlinear, highly coupled, time-dependent and inherently unstable,

\footnotetext{
a herwin.suprijono@ui.ac.id
}

and those parameters may varies widely across the full flight condition in its course. Some researchers have designed and constructed the controller of the helicopter using mathematical modelling, but unfortunately, there are many complexities that cannot be modelled precisely due to various assumptions. When the helicopter flew vertically, for example, errors may arise due to the simplification of the model by ignoring the nonlinearity effects that usually occurs in the actuator kinematic, ground effect, servo dynamics, rotor speed variation, sensor lag, and rotor inflow lag, which is associated with the rate of change of blade pitch. Another approach for the RUAV controller is developed based on non-linear control methods including a back-stepping method [1-3], fuzzy logic [4-6], sliding mode control [7], and LQR control [8].

Recently, neural networks controller has been developed and applied in several flight control applications [9-13]. The development of neural networks as a control system is driven by a requirement of using an adaptive controller when a specified plant is contained within uncertain conditions, without the need for tuning offline. Nowadays a more efficient algorithm has been developed based on the identification of nonlinear system using a multilayer feedforward neural network with backpropagation learning and its variations. Neural networks are able to identify and control the dynamic so that it was chosen for modelling the non-linear systems using multi-layer neural network approximation. A direct inverse control (DIC) technique using a neural networks architecture is chosen as the controller in this paper because of its simplicity to implement within the constraints of the flight control computer software, easy to be optimized optimization the control, including when 
hovering and maneuvering, Compare to that of conventional controller system, neural networks based DIC technique used more less memory requirement, less computational time, and the most important is that a better control of the flight behaviour could be achieved.

\section{Helicopter and avionic system}

In this research a small UAV helicopter TREX 450 is utilized, which has some unique advantages compared with the larger counterparts, including: 1) more agility; 2) easier to assemble and move; 3 ) less noise; 4) lower cost and 5) more suitable for forest, urban searching and indoor flight. Due to its smaller size, however, extremely strict payload and more sensitive aerodynamics maneuvers are necessary, and the problem on constructing a small UAV helicopter is much more difficult and more challenging. TREX 450 is equipped with one pair of stabilizer bar to dampen the over drastic aerodynamics due to its small size, and a yaw rate gyro which facilitates the manual controller to realize the yaw rate stabilization. The swash-plate is used to translate the command of the pilot into the propeller movement and the stabilized bar by changing the main motor speed. Compared with the larger counterparts who are normally powered by nitro-fuel or gasoline, the small UAV TREX 450 has a reduce vibration level due to electrically powered servo motors. The TREX 450 flying maneuvers is performed by five servo actuators; three servos are connected to the swash-plate through a collective cyclic field mixing method (CCFM), which collectively actuating the aileron and the elevator. Each servo and the BLDC as the main motor are controlled by using a Pulse Width Modulation (PWM) system with a rate of $50 \mathrm{~Hz}$.

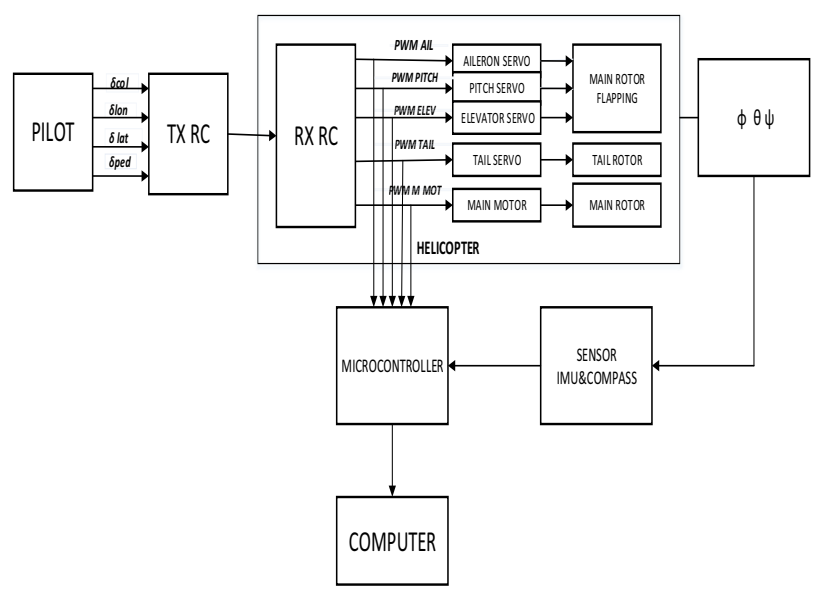

Figure 1. Avionic System

Block diagram of the small UAV and its avionic system is depicted in Figure 1. As can be seen clearly in the avionics architecture of the UAV system, the PWM signals from the receiver are used to move the actuators which make the UAV movements, and the data from the Inertial Measurement Unit (IMU) for monitoring the UAV conditions are read by avionics interface section. The IMU used in this avionic system consists of a digital compass HMC 5883L chip, a 6 DOF accelerometer, a Gyro MPU6000 and a barometric pressure sensor; while the main component of the avionics interface unit is a microcontroller AT Mega 2560. Measurement data from the IMU and PWM signal for each servo are sent to the Ground Station system through the RS 232 serial interface with a speed of $115.200 \mathrm{bps}$. The Ground Station system is composed of a laptop with Windows 7 operating system and a LABVIEW software that is utilized such that the flight data along its movement can be collected and displayed in a real time fashion.

\section{Neural network direct inverse control (NN-DIC)}

The NN-DIC algorithm is a novel concept based on the Neural Networks control using the inverse of the plant as the model of controller. The configuration of the NN-DIC algorithm is shown in Figure 2 below. The inverse model of the plant is represented by neural networks and should be trained until a predetermined minimum error is achieved before it is implemented as a controller. The method which usually used training mechanism for this supervised direct inverse model is the backpropagation learning method.

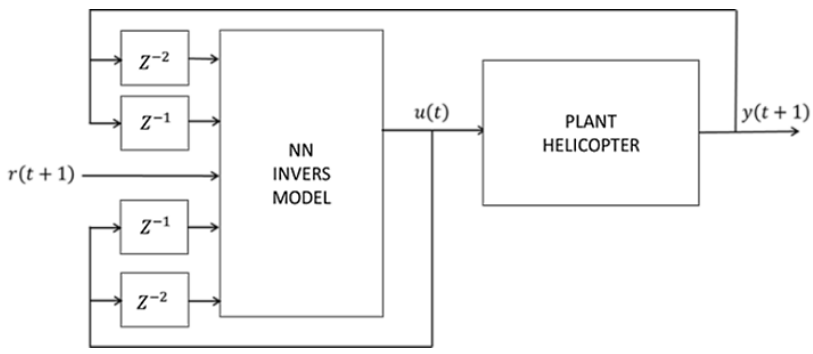

Figure 2. Direct Inverse Structure

The required training data for training the neural networks are collected from the TREX 450 small helicopter through a human operator using a remote control system to make various maneuvers of the helicopter within a number of experimental flights. The TREX450 flights data that are collected from each experimental flight contains the PWM signal to each servo, the attitude data of roll, pitch, yaw, and the altitude of helicopter in degrees. The sampling time for those data acquisition system is determined to be $200 \mathrm{msec}$ and 1000 data sample are collected and automatically stored in the Ground Station system.

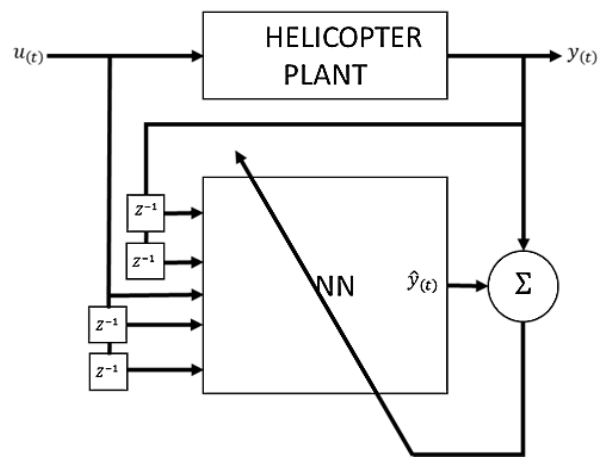

Figure 3. Block diagram Identification 


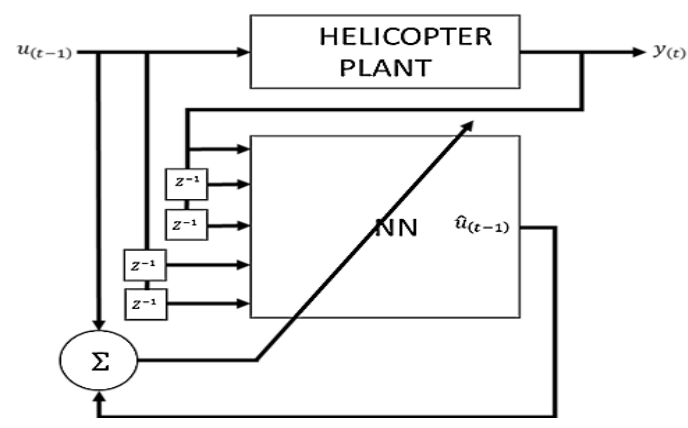

Figure 4. Block diagram Inverse

The required data for training the neural networks are collected from the TREX 450 small helicopter through a human operator using a remote control system to make various maneuvers of the helicopter within a number of experimental flights. The TREX450 flights data that are collected from each experimental flight contains the PWM signal to each servo, the attitude data of roll, pitch, yaw, and the altitude of helicopter. The sampling time for those data acquisition system is determined to be $200 \mathrm{mS}$. 1000 data sample are collected and automatically stored in the Ground Station system.

\section{Experiment Result}

For the purpose of this research is to identify a Neural Networks model for the TREX 450 small helicopter, in this case, the configuration for training is shown in figure 3 , where the Neural Networks has 26 input neurons, 4 output neurons and 35 neurons in the hidden layers. The output vector of the neural networks consists of the roll, pitch, yaw and altitude, and by comparing with the actual output data output from the plant $y(t)$, the error signal is used to train the networks. The 1000 data sample that is collected along the flight experiment are used to trained the $\mathrm{NN}$ using backpropagation method, and after 140.000 iterations, the MSSE is $5.5 \times 10^{-5}$. Comparison results of the output signal and the actual output signal for the roll, pitch, yaw and altitude are shown in Figure 5. It is clearly seen from this figure that these $\mathrm{NN}$ output signals are almost identical with the actual flight data.
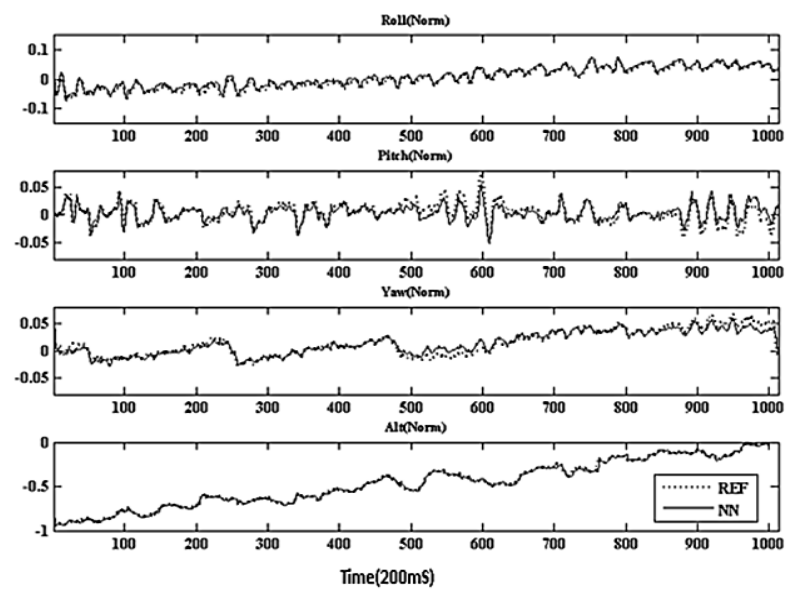

Figure 5. Comparison of $y(t)$ and $\hat{y}(t)$
To obtain the inverse model of the UAV, the configuration setup as shown in Figure 4 is used. The architecture of the neural networks as the inverse model consists of an input layer with 24 neurons, a single hidden layer with 36 neurons and an output layer with 6 neurons. The output vector of the $\mathrm{NN}$ inverse model represents the 6 PWM signals which are necessary to control the servo motors. The neural networks inverse is trained by using backpropagation method in order to obtain the minimum error signal defined by $e(t)=u(t)-\hat{u}(t)$. In each iterations (epoch), 1000 data sample are used, and after 177.000 epochs, the learning system reaches the minimum error MSSE of $1.103 \times 10^{-4}$. Results of the output signal comparison between the actual control signal of the UAV, $u(t)$, and the neural networks inverse model output, $\hat{u}(t)$, are depicted in Figure 6. As can be seen from this figure, these signals are almost identical with a minimum error of MSSE equal to $1.103 \times 10^{-4}$, nearly the same with that of the neural networks inverse model when in the learning stage.
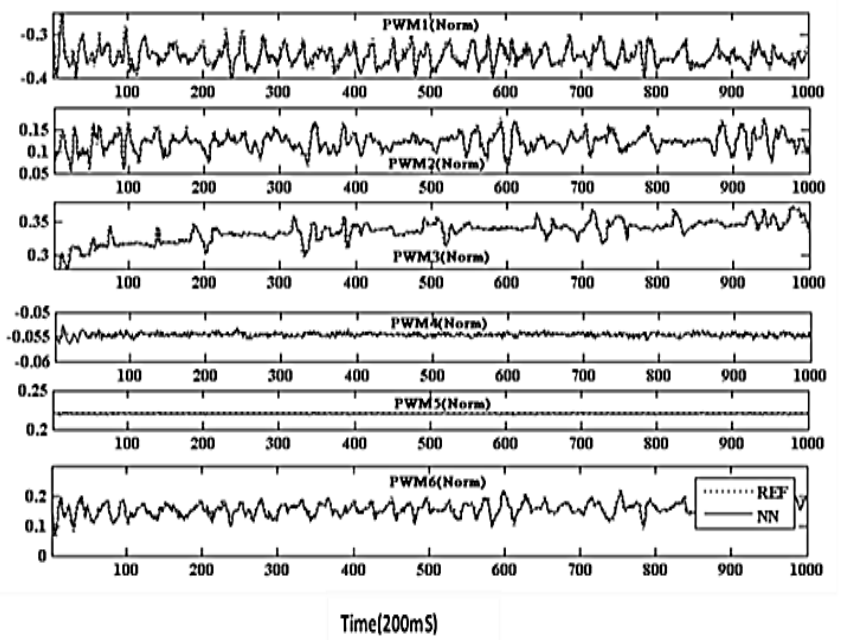

Figure 6. Comparison of $u(t)$ and $\hat{u}(t)$

Finally, the direct inverse neural networks model is cascading with the system identification neural networks of the UAV plant to provide an identity mapping between the signal reference and the output signal of the UAV. Figure 2 shows the close-loop cascading the NN direct inverse model and the $\mathrm{NN}$ identified model of the TREX450 helicopter for the purpose of simulation. In this experiment, we set the reference signal as follows: roll parameter, pitch parameter and yaw parameter is determined at $0^{\circ}$, while altitude is $250 \mathrm{~cm}$. This situation represents the simulation of hovering condition of the helicopter at an altitude of $250 \mathrm{~cm}$. The result of the control experiment is shown in Figure 7. As can be seen from this figure, all of the output signals are converging to the reference set points after around 20 to 25 time steps with a sampling time of $200 \mathrm{mS}$.

In order to have a better performance of the direct inverse controller, an optimization scheme is proposed by re-training the $\mathrm{NN}$ inverse model using the new data. The optimization training is done just for only 3.000 epochs more, however, the achieved MSSE after it is converging is $8.35 \times 10^{-5}$. The optimized direct inverse is then put back 
into the cascade direct inverse system and the simulation simulation results of the experiments are shown in Figure 8.
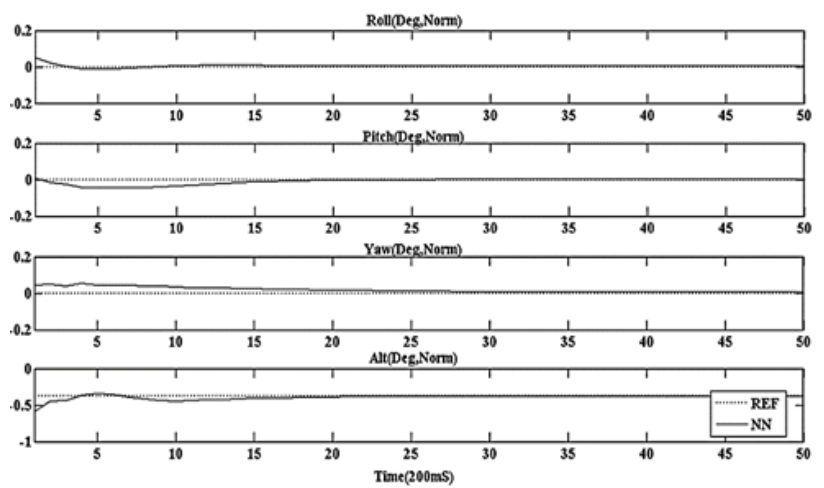

Figure 7. Result of the used Direct Inverse Control without optimization method
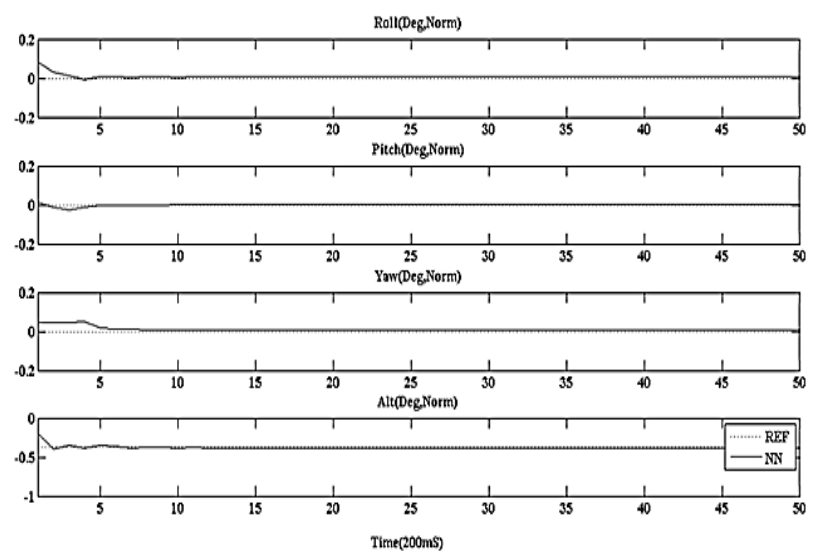

Figure 8. Experiment results after optimization by re-training

As can be seen from this figure, all of the output signals are converging to the reference set points is around 5-10 times steps, which indicating a much faster convergence compare with that of the un-optimized direct inverse controller.

\section{Conclusion}

We have developed a neural networks direct inverse controller for a small helicopter UAV system. To obtain a better performance of the DIC model, an optimization scheme is proposed, and experimentally compare with that of the un-optimized DIC model. The experimental results show that both of the neural networks DIC model and the optimized neural networks DIC model can stabilize the helicopter with good tracking performance. Results also show that the optimized DIC improved the performance characteristics of the UAV in maintaining a hover condition.

\section{References}

1. Zhu Bing, Huo Wei, "Adaptive backstepping control for a miniature autonomous helicopter," Decision and Control and European Control Conference, 2011 50th IEEE Conference, pp.5413-5418, (2011).
2. Roy T.K., Suman A.A., "Adaptive backstepping controller for altitude control of a small scale helicopter by considering the ground effect compensation," Informatics, Electronics \& Vision (ICIEV), pp.1-5, (2013).

3. B. Ahmed, H. R. Pota, and M. Garratt, "Flight control of a rotary wing UAV using backstepping," International Journal of Robust and Nonlinear Control, vol. 20, pp. 639-658, (2010).

4. Sasaki. M., Ishida. H., Katsuno. T.; Ogasawara, A., "Learning fuzzy logic controller for hovering a helicopter," Fuzzy Information Processing Society NAFIPS, pp.25-28, (1998).

5. Velagic. J, Osmic. N, "Design and implementation of fuzzy logic controllers for helicopter elevation and azimuth controls," Control and Fault-Tolerant Systems (SysTol), pp.311-316, (2010).

6. Xin Hou, Ping Li, Zhou Fang; Bo Han, "An Application of Fuzzy PID Algorithm on Unmanned Helicopter Attitude Control", Intelligent Control and Automation, 2006. WCICA 2006. The Sixth World Congress on, vol.2, pp.9129-9133, (2006).

7. Woonchul Ham, Hyeokjae Kwon, "Sliding mode control for the hovering of helicopter by using quaternion dynamics," SICE Annual Conference (SICE), pp.1024-1028, (2011).

8. Chen You, Wang Xiafu, Lu Geng, Zhong Yisheng, "Modeling and LQR control of small unmanned helicopter," Control Conference (CCC),pp.43014305,(2013).

9. Doohwan Park, Joontark Lee, Honggon Ha, "Attitude control of helicopter simulator using neural network based PID controller," Fuzzy Systems Conference Proceedings, 1999. FUZZ-IEEE '99, pp.465- 469 vol.1, (1999).

10. Frye, M.T, Provence R.S, "Direct Inverse Control using an Artificial Neural Network for the Autonomous Hover of a Helicopter," Systems, Man and Cybernetics (SMC), 2014 IEEE International Conference, pp.4121-4122, (2014).

11. M. Garratt and S. Anavatti, "Non-linear Control of Heave for an Unmanned Helicopter Using a Neural Network," Journal of Intelligent \& Robotic Systems, vol. 66, pp. 495-504, (2012).

12. Samal M, Sreenatha A. G, \& Garratt M," Neural network based system identification for autonomous flight of an eagle helicopter", IFAC), pp. 7421-7426, (2008).

13. Buskey G, Wyeth G, \& Roberts J. "Autonomous helicopter hover using an artificial neural network". Proceedings 2001 ICRA. IEEE International Conference on Robotics and Automation, 1-7, (2001). 